\title{
Dexmedetomidina epidural em gatas submetidas à ovariosalpingohisterectomia sob anestesia total intravenosa com propofol e pré-medicadas com cetamina $\mathrm{S}(+)$ e midazolam
}

\author{
Epidural dexmedetomidine in cats submitted to ovariosalpingohisterectomy under intravenous total \\ anesthesia with propofol and pre medicated with ketamine (S) and midazolam
}

\author{
Otávia Dorigon $^{\mathrm{I}}$ Nilson Oleskovicz* ${ }^{\mathrm{I}}$ Aury Nunes de Moraes ${ }^{\mathrm{I}}$ Ademar Luiz Dallabrida ${ }^{\mathrm{I}}$ Fabíola \\ Niederauer Flôres ${ }^{I}$ André Vasconcelos Soares ${ }^{\mathrm{II}}$ Thiago José Mores ${ }^{\mathrm{III}}$
}

RESUMO

O objetivo deste estudo foi avaliar os efeitos da administração epidural de dexmedetomidina em gatas submetidas à ovariosalpingohisterectomia (OSH), sob infusão contínua de propofol. Para tanto, foram utilizadas 12 gatas

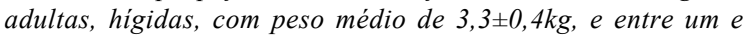
quatro anos de idade. Como medicação pré-anestésica, os animais receberam cetamina $\mathrm{S}(+) \quad\left(5 \mathrm{mg} \mathrm{kg}^{-1}\right) \quad e$ midazolam( $\left.0,5 \mathrm{mg} \mathrm{kg}^{-1}\right)$, pela via intramuscular, e propofol como agente indutor $\left(4 \mathrm{mg} \mathrm{kg}^{-1}\right)$, pela via intravenosa. Em seguida, os animais foram intubados para receber oxigenio a $100 \%$, por meio de sistema sem reinalação de gases, e a infusão contínua de propofol $\left(0,3 \mathrm{mg} \mathrm{kg}^{-1} \mathrm{~min}^{-1}\right)$ foi iniciada. Os animais foram alocados em dois grupos, nos quais foram administrados dexmedetomidina $\left(2 \mu \mathrm{g} \mathrm{kg}^{-1}\right)$ por via epidural $(G D E X, n=06)$ e, no grupo controle (GSAL, $n=06)$, solução salina $0,9 \%$ pela mesma via. $O$ volume total da solução para administração epidural foi completado com solução salina e ajustado para $0,26 \mathrm{ml} \mathrm{kg}^{-1}$ em ambos os grupos. No GSAL, houve necessidade de administração de fentanil imediatamente após o início da cirurgia (T10) em 33,33\% dos pacientes. Durante a infusão contínua de propofol, o reflexo palpebral medial esteve presente em 66,6\% dos animais do GSAL e em $16,6 \%$ dos animais do GDEX. O globo ocular permaneceu centralizado em todos os animais do GSAL e rotacionado em $83,4 \%$ dos animais do GDEX. Foi observada redução da freqüência cardíaca após a indução anestésica em ambos os grupos. A pressão arterial média foi menor no T0 e no T5, no $G D E X$, e maior no T10, no GSAL, em relação ao basal. A freqüencia respiratória foi menor no GDEX nos tempos $T 20$ e $T 30$ em relação a GSAL. A $\mathrm{SaO}_{2}$ e a $\mathrm{PaO}_{2}$ foram maiores de $T 5$ até T40 para ambos os grupos em comparação ao basal. Para a $\mathrm{PaCO}_{2}$, foram observados valores maiores de T5 até T40 para o GDEX e maiores no T5, T10 e T20, no GSAL, em relação aos valores basais. Na recuperação anestésica, $50 \%$ e $16,6 \%$ dos animais apresentaram agressividade no GSAL e $G D E X$, respectivamente. Com relação à intensidade de dor ao toque da ferida, foi observado que 33,3\% dos animais do GSAL e 66,7\% dos animais do GDEX receberam escore 0 (sem reação). Conclui-se que os animais que receberam administração de dexmedetomidina pela via epidural apresentaram plano de anestesia mais estável com maior grau de analgesia no periodo trans e pós-operatório, recuperação anestésica de melhor qualidade, sem alterações cardiovasculares e hemogasométricas significativas.

Palavras-chave: gatos, epidural, dexmedetomidina, propofol.

\section{ABSTRACT}

The objective of this study was to evaluate the effect of the epidural administration of dexmedetomidine in cats submitted to ovariohisterectomy, under continuous infusion of propofol. The cats received $S(+)$ ketamine $\left(5 \mathrm{mg} \mathrm{kg}^{-1}\right)$ and midazolan $\left(0.5 \mathrm{mg}^{\mathrm{kg}^{-1}}\right)$ as premedication by intramuscular injection, and propofol as induction agent $\left(4 \mathrm{mg} \mathrm{kg}^{-1}\right)$, by intravenous injection. After that, the cats were intubated and kept on oxygen $100 \%$. Subsequently the continuous infusion of propofol was initiated $\left(0.3 \mathrm{mg} \mathrm{kg}^{-1} \mathrm{~min}^{-1}\right)$. The animals were divided into two groups, in the dexmedetomidine group (DEXG, $n=6,2 \mu \mathrm{g} \mathrm{kg}^{-1}$ ) dexmedetomidine was administered by epidural injection, and in the control group (SALG, n=6) saline solution was administered by the same way. In both groups the final volume was $0.26 \mathrm{~mL} \mathrm{~kg}^{-1}$ completed with $\mathrm{NaCl} 0.9 \%$. Fentanyl administration was necessary at T10 in $33.3 \%$ of the patients. During the continuous infusion of propofol, the medial palpebral reflex was present in $66.6 \%$ of the animals of $S A L G$ and in $16.6 \%$ of the animals of DEXG. The eyeball was centralized in all the animals of SALG and $83.4 \%$ of the animals of DEXG

IDepartamento de Medina Veterinária, Centro de Ciências Agroveterinárias, Universidade do Estado de Santa Catarina (UDESC). Av. Luiz de Camões, 2090, Conta Dinheiro, 88520-000, Lages, SC, Brasil. E-mail: a2no@cav.udesc.br. *Autor para correspondência. "Programa de Pós-graduação em Medicina Veterinária, Universidade Federal de Santa Maria (UFSM), Santa Maria, RS, Brasil. IIIPrograma de Pós-graduação em Ciências Veterinárias,Universidade Federal do Rio Grande do Sul (UFRGS), Porto Alegre, RS, Brasil. 
the eyeball were with ventral rotation. Reduction of the heart rate was observed after anesthesia induction in both groups. The mean arterial pressure was significantly lesser at TO and T5 in the DEXG and greater at T10 in the SALG when compared to the basal values. The respiratory rate was lesser in the DEXG at T2O and T30 when compared to SALG. The $\mathrm{SaO}$, and $\mathrm{PaO}$ were bigger from $\mathrm{T5}$ to T40. The $\mathrm{PaCO}_{2}$ bigger values were observed at T5, T10, T20, T30 and T40 for the DEXG and at T5, T10 and T20 in the SALG when compared to the basal values. In the anesthetic recovery, $50 \%$ and $16.6 \%$ of the animals presented aggressiveness in the SALG and DEXG, respectively. To the wound pain threshold, it was observed that $33.3 \%$ of animals of SALG and $66.7 \%$ of the animals of DEXG received score 0 (without reaction). It was concluded that the animals that received dexmedetomidine administration by epidural injection, presented more stable anesthetic plan with greater analgesia degree in the peri and postoperative period, better quality anesthesia recovery, and without significant cardiovascular and hemogasometric changes.

Key words: cats, epidural, dexmedetomidine, propofol.

\section{INTRODUÇÃO}

Os felinos possuem diversas particularidades em relação à metabolização de fármacos, com respostas clínicas específicas a alguns agentes (ILKIW et al., 1996). A utilização de protocolos intravenosos injetáveis tem sido amplamente utilizada na anestesia atualmente (SELMI et al. 2005). A anestesia intravenosa total (AIT) pode ser definida como uma técnica que utiliza fármacos administrados pela via intravenosa para produzir inconsciência, analgesia e relaxamento muscular, com depressão cardiorrespiratória e efeito cumulativo mínimo (CASTRO, 2005). Acredita-se que, em alguns protocolos, esse tipo de técnica produza efeitos cardiovasculares menos pronunciados que o emprego da anestesia geral inalatória. SELMI et al. (2005) demonstraram que o emprego de propofol na dose de $0,3 \mathrm{mg} \mathrm{kg}^{-1} \mathrm{~min}^{-1}$ produz anestesia satisfatória, com redução da freqüência cardíaca (FC), pressão arterial (PA) e depressão respiratória em gatos pré-medicados com a associação de cetamina-midazolam.

A administração conjunta de cetamina e benzodiazepínicos é uma das associações mais empregadas tanto como medicação pré-anestésica (SELMI et al., 2005), quanto anestesia de pequenos animais, proporcionando segurança, mínimos efeitos depressores, além de rápida recuperação anestésica (HELLYER et al., 1991; ILKIW et al., 1998). O midazolam é um benzodiazepínico que produz ligeira queda da pressão arterial (PA), com boa potência hipnótica (CORTOPASSI \& FANTONI, 2002). A cetamina, por aumentar a FC, o débito cardíaco (DC), a PA, o trabalho do ventrículo esquerdo e o consumo de oxigênio pelo miocárdio (LIN, 1996), tem sido associada a sedativos ou tranqüilizantes que eliminam ou minimizam esses efeitos (HELLYER et al., 1991).

O propofol é um anestésico geral, rapidamente metabolizado (PADDLEFORD, 2001) em gatos, que promove indução e recuperação anestésicas suaves (PASCOE et al, 2006); porém, produz queda da PA e depressão respiratória (MUIR \& GADAWSKI, 2002). O fígado do gato não biotransforma os fenóis de forma rápida como o fígado do cão, pois apresenta menor capacidade de glucoronização (ROBERTSON, 2005).

O controle da dor trans e pós-operatória ou pós-traumática tem sido uma das grandes preocupações dos anestesistas e clínicos de pequenos animais. Nesse contexto, destaca-se a anestesia epidural, que é utilizada para controle da dor abdominal e de membros pélvicos e tem como principais vantagens a segurança, a eficiência e o baixo custo. A anestesia epidural, com anestésicos locais, possui baixa seletividade, produzindo tanto bloqueio sensitivo, quanto bloqueio motor, tornando essa técnica pouco atrativa para o controle da dor por períodos prolongados (TORSKE et al., 1998). Nesse sentido, seria possível empregar os agonistas alfa adrenérgicos, pois estes produzem analgesia, por ativação colinérgica espinhal, sem causar bloqueio motor (EISENACH et al., 1996). A dexmedetomidina é um enantiômero da medetomidina e, quando administrada na metade da dose, promove efeitos farmacológicos similares (OTERO, 2005). Possui alta seletividade para os receptores alfa $_{2}$, promove boa estabilidade hemodinâmica e reduz o requerimento de anestésicos durante a anestesia (VILLELA \& JUNIOR, 2003). SOUZA (2006) observou que a administração epidural e a infusão contínua intravenosa de dexmedetomidina reduziram o consumo do agente inalatório e produziram recuperação de melhor qualidade e mais prolongada em relação ao grupo que recebeu somente lidocaína pela via epidural. Segundo VILLELA\& JUNIOR (2003) e BAGATINI et al. (2002), a dexmedetomidina produz efeitos cardiovasculares semelhantes aos demais agonistas alfa2 adrenérgicos, não promovendo depressão respiratória importante ou depressão nos valores de gases sangüíneos.

Em virtude da escassa literatura, o objetivo deste estudo foi avaliar os efeitos cardiorespiratórios, hemogasométricos e analgésicos da dexmedetomidina, pela via epidural, associada à anestesia intravenosa total com propofol, após medicação pré-anestésica com cetamina $\mathrm{S}(+)$ e midazolam, em gatas submetidas à ovariosalpingohisterectomia. Além disso, foram avaliados aspectos clínicos, como qualidade de 
indução e intubação, grau de sedação, qualidade do plano anestésico (relaxamento muscular, presença de reflexo palpebral medial e lateral e posição do globo ocular) e período de recuperação imediato (alteração comportamental, intensidade de dor ao toque da ferida cirúrgica, $\mathrm{FC}, f \mathrm{e}$ TR).

\section{MATERIAL E MÉTODOS}

Foram utilizadas 12 gatas adultas, hígidas, com peso médio de $3,3 \mathrm{~kg} \pm 0,4$, e entre um e quatro anos de idade. Os animais foram manejados e condicionados ao ambiente de experimentação uma vez ao dia, durante 30 dias. Após esse período, eles foram submetidos a jejum sólido de 12 horas e hídrico de seis horas. Os animais foram anestesiados, durante 10 minutos, com sevoflurano ${ }^{\mathrm{a}} 5 \mathrm{~V} \%$, administrado por máscara facial com fluxo diluente de oxigênio de $4 \mathrm{~L} \mathrm{~min}^{-1}$. Em seguida, foi introduzido e fixado um cateter $22 \mathrm{G}$ na veia cefálica, utilizado para administração do propofol, e foi dissecada a artéria femural direita, para introdução e fixação de um cateter de polietileno $(22 \mathrm{G})$, para mensuração da PA e obtenção das amostras de sangue para hemogasometria. Após a instrumentação, a administração de sevoflurano foi encerrada, e um período de 30 minutos foi aguardado para a recuperação dos animais.

As gatas receberam como medicação pré anestésica (MPA) cetamina $\mathrm{S}(+)^{\mathrm{b}}\left(5 \mathrm{mg} \mathrm{kg}^{-1}\right)$ associada ao midazolam ${ }^{\mathrm{c}}\left(0,5 \mathrm{mg} \mathrm{kg}^{-1}\right)$, pela via intramuscular. Após 15 minutos, foi administrado propofol ${ }^{\mathrm{d}}\left(4 \mathrm{mg} \mathrm{kg}^{-1}\right)$, pela via intravenosa, foi realizada a intubação orotraqueal e foi fornecido oxigênio a $100 \%$ por meio de sistema sem reinalação de gases durante o período de manutenção anestésica. Subseqüentemente, foi iniciada a infusão contínua de propofol ${ }^{\mathrm{d}}\left(0,3 \mathrm{mg} \mathrm{kg}^{-1} \mathrm{~min}^{-1}\right)$, diluído em solução salina $0,9 \%$, na dose de $10 \mathrm{~mL} \mathrm{~kg}^{-1} \mathrm{~h}^{-1}$, e administrado por meio de bomba de injeção de seringa.

Os animais foram alocados em dois grupos e receberam, pela via epidural, dexmedetomidina ${ }^{\mathrm{e}}(2 \mu \mathrm{g}$ $\left.\mathrm{kg}^{-1}\right)(\mathrm{GDEX}, \mathrm{n}=06)$ e solução salina $0,9 \%(\mathrm{GSAL}, \mathrm{n}=06)$. $\mathrm{O}$ volume total da solução administrada foi ajustado para $0,26 \mathrm{ml} \mathrm{kg}$ em ambos os grupos. O posicionamento adotado para realização da anestesia epidural lombosacra foi o decúbito esternal, com os membros pélvicos flexionados cranialmente. A correta localização da agulha no espaço epidural foi confirmada pela ausência de resistência à aplicação de ar com auxílio de uma seringa de vidro (SKARDA, 1996). Em seguida, os animais foram posicionados em decúbito dorsal, com a cabeça no mesmo plano do corpo e, após cinco minutos, foi iniciado o procedimento cirúrgico, o qual foi padronizado em 30 minutos até a sutura de pele.
Foram avaliados os seguintes parâmetros: freqüência cardíaca (FC), freqüência respiratória (f), temperatura retal (TR), pressão arterial sistólica (PAS), pressão arterial média (PAM) e pressão arterial diastólica (PAD), hemogasometria arterial e eletrólitos $\left(\mathrm{SaO}_{2}, \mathrm{PaO}_{2}, \mathrm{PaCO}_{2}, \mathrm{pH}, \mathrm{Ca}^{+}, \mathrm{Na}^{+}, \mathrm{K}^{+}\right.$, déficit de base (DB) e bicarbonato), relaxamento muscular (0 sem relaxamento; 1 leve; 2 moderado, 3 ótimo), reflexo palpebral lateral (RPL) e medial (RPM) (ausente/ presente), posição do globo ocular (GO) (0 centralizado; 1 levemente rotacionado; 2 rotacionado) e grau de sedação ( 0 sem sedação; 1 leve; 2 ótima) nos seguintes tempos: T-15 (basal), T0 (15 minutos após a administração da MPA), T5 (após o início da infusão contínua), T10, T20, T30, T40 (10, 20, 30, 40 minutos após a anestesia epidural). Foram avaliados também os seguintes parâmetros: a qualidade de indução (suave ou com reação (excitação)), a qualidade de intubação (suave ou com dificuldade), a qualidade de recuperação (suave ou com excitação) e o tempo de extubação (minutos). Considerou-se como resposta dolorosa positiva, o aumento de FC ou PAS superior a $20 \%$ do valor referente ao momento anterior. Nesse caso, foi administrada dose complementar de analgésico (fentanil ${ }^{\mathrm{f}} 4,4 \mathrm{mcg} \mathrm{kg}^{-1}$ pela via intravenosa).

No período pós-operatório imediato, foi avaliada a alteração comportamental (presença ou não de agressividade), intensidade de dor ao toque da ferida cirúrgica ( 0 sem reação; 1 reação leve; 2 reação intensa), $\mathrm{FC}, f$, TR em intervalos de uma hora, durante seis horas. Como medicação pós-operatória, foi administrada enrofloxacina $\left(5 \mathrm{mg} \mathrm{kg}^{-1}\right)$, pela via intramuscular, uma vez ao dia, durante quatro dias. Os dados paramétricos foram comparados entre os grupos pelo teste $t$, e as diferenças entre os tempos dentro de cada grupo foram analisadas pela Análise de Variância com Repetições Múltiplas. As diferenças observadas foram analisadas pelo teste de Student-Newman-Keuls $(\mathrm{P} \leq 0,05)$.

\section{RESULTADOS E DISCUSSÃO}

O grau de sedação após a MPA foi considerado ótimo em $83,4 \%$ e leve em $16,6 \%$ dos animais, para ambos os grupos. Esses dados estão de acordo com os obtidos por SELMI et al. (2005), os quais observaram sedação e decúbito após a administração de cetamina ( $\left.3 \mathrm{mg} \mathrm{kg}^{-1}\right)$ e midazolam $\left(0,3 \mathrm{mg} \mathrm{kg}^{-1}\right)$ em $50 \%$ dos gatos avaliados.

A indução anestésica foi suave em todos os animais do GDEX, e no GSAL foi considerada suave em $83,4 \%$, com presença de dor em 16,6\% dos animais. A qualidade de intubação foi suave em $83,4 \%$ e com dificuldade em $16,6 \%$ dos animais. BOUILLON et al. 
(2004) afirmam que a excitação e as respostas autônomas à laringoscopia e intubação traqueal poderiam ser anuladas pela combinação de fármacos hipnóticos e analgésicas durante a indução. SELMI et al. (2005) observaram indução anestésica desprovida de incidentes adversos (apnéia, excitação, êmese) em gatos após a administração de propofol com agente indutor único. Em contrapartida, FUJII \& ITAKURA (2008), HONARMAND \& SAFAVI (2008) e KAYA et al. (2008) afirmam que o propofol em macroemulsão produz dor no local de aplicação, podendo ocasionar resposta adversa ou reação do paciente no momento da aplicação, tornando-se necessária a aplicação concomitante de outro fármaco para minimizar ou anular esse efeito.

Durante a infusão contínua de propofol, o RPL foi ausente em todos os animais no GDEX e GSAL. O RPM esteve presente em 66,6\% (4/6) dos animais do GSAL e em 16,6\% (1/6) dos animais do GDEX. Em relação ao GO, todos os animais do GSAL apresentaram o globo ocular centralizado, enquanto que, no GDEX, $83,4 \%(5 / 6)$ apresentaram o globo rotacionado. Com base na posição do $\mathrm{GO}$, dos reflexos palpebrais (medial e lateral) e do relaxamento muscular, foi observado que os animais do GDEX apresentaram plano anestésico mais profundo em virtude do efeito sedativo aditivo da dexmedetomidina. Isso pode ser comprovado pela necessidade de administração de fentanil $\left(4,4 \mathrm{mcg} \mathrm{kg}^{-1}\right)$ no início do procedimento cirúrgico entre T5 e T10 em $33,3 \%$ (2/6) dos animais do GSAL. Nesse sentido, vários estudos demonstram que os alfa -agonistas reduzem o requerimento anestésico. Em cães, a dexmedetomidina reduziu a concentração alveolar mínima (CAM) do isoflurano em 86\% (WEITZ et al. 1991), 89\% (BLOOR et al., 1992) e 60\% (PASCOE et al., 2006).

Todos os animais do GSAL e GDEX apresentaram relaxamento muscular ótimo (grau 3), sendo este considerado adequado para realização do procedimento cirúrgico. Esses dados comprovam que a adição de dexmedetomidina epidural não aumentou o relaxamento muscular produzido pelo propofol ou pela medicação pré-anestésica. Esses dados corroboram os resultados de SELMI et al (2005), os quais afirmam que a AIT com propofol é uma técnica aceitável para manutenção da anestesia e que o emprego de cetamina $\left(3 \mathrm{mg} \mathrm{kg}^{-1}\right)$ e midazolam $\left(0,3 \mathrm{mg} \mathrm{kg}^{-1}\right)$, pela via intramuscular, seguido do emprego de propofol $(0,3 \mathrm{mg}$ $\mathrm{kg}^{-1} \mathrm{~min}^{-1}$ ), produz anestesia satisfatória.

Não foram observadas diferenças significativas para PAS, PAM, PAD, TR, $\mathrm{SaO}_{2}, \mathrm{PaO}_{2}$, $\mathrm{K}^{+}$, hematócrito, $\mathrm{HCO}_{3}^{-}$entre os dois grupos.

Houve redução da PAS imediatamente após o início da infusão de propofol (T5) no GDEX e aumento logo após o início da cirurgia (T10) no GSAL. A PAM foi menor após a MPA (T0) e após o início da infusão de propofol (T5) no GDEX, em relação aos valores basais, e maior logo após o início da cirurgia (T10) no GSAL (Tabela 1). Esse aumento da PAM no GSAL foi considerado como resposta de dor, sendo administrado imediatamente fentanil, na dose de $4,4 \mathrm{mcg} \mathrm{kg}^{-1}$, em $33,3 \%(2 / 6)$ dos animais do GSAL. A PAD foi menor após o início da infusão de propofol (T5) no GDEX. De acordo com YANG et al. (1997), a vasodilatação periférica, redução do tônus simpático, ação cronotrópica e inotrópica negativas e depressão do reflexo barorreceptor são os principais fatores responsáveis pela redução da PA após a administração de propofol.

Clinicamente foi observada redução gradativa da FC no GDEX a partir de T20. Em T40, essa redução foi de $20,75 \%$, em comparação com T-30, diferindo dos valores do GSAL nesse momento. Esses resultados estão de acordo com os obtidos por FUKUSHIMA et al. (1997), os quais observaram que a administração epidural de $2 \mathrm{mcg} \mathrm{kg}^{-1} \mathrm{de}$ dexmedetomidina em mulheres submetidas à histerectomia propiciou a redução da FC em 25\%, da PA em $20 \%$ e dos níveis plasmáticos de adrenalina e noradrenalina em $30 \%$. Os resultados obtidos no presente estudo diferem de SELMI et al. (2005), os quais observaram redução significativa da FC após a infusão de $0,3 \mathrm{mg} \mathrm{kg}^{-1} \mathrm{~min}^{-1}$ de propofol em gatos.

A $\mathrm{SaO}_{2}$ e a $\mathrm{PaO}_{2}$ foram maiores após a intubação (T5) até o final do período de avaliação (T40) em ambos os grupos (Tabela 1). Esses dados são semelhantes aos descritos por GABAS et al. (2006), pois, nesse período, os animais mantiveram-se intubados e com suplementação de oxigênio durante o procedimento cirúrgico.

A f foi menor no GDEX nos tempos T20 e T30 em relação ao GSAL. Entre os tempos, foram observados valores menores de T5 até T40, no GDEX, e de T5 até T30, no GSAL, em relação aos valores basais (Tabela 1). Para a $\mathrm{PaCO}_{2}$, foram observados valores maiores desde o início da infusão de propofol (T5) até o período final de avaliação (T40), para o GDEX, e maiores de T5 até T20, no GSAL, em relação aos valores basais. Para o $\mathrm{pH}$, os valores foram menores de T5 até T40 no GDEX e menores de T5 até T30 no GSAL. Segundo KUUSELA et al. (2001), a dexmedetomidina administrada em cães diminui a f, o volume corrente, a $\mathrm{PaO}_{2}$, o pH e os níveis de bicarbonato sanguíneo, além de aumentar a $\mathrm{PaCO}_{2}$ de forma dose-dependente. $\mathrm{O}$ propofol induz depressão respiratória significativa por ação direta sobre os centros respiratórios e a resposta ventilatória à $\mathrm{PaCO}_{2}$, sendo bastante pronunciada 
Tabela 1 - Valores médios e desvio padrão da pressão arterial média (PAM), freqüência cardíaca (FC), freqüência respiratória (f), pressão de dióxido de carbono $\left(\mathrm{PaCO}_{2}\right)$, pressão parcial de oxigênio arterial $\left(\mathrm{PaO}_{2}\right)$, saturação de oxigênio na hemoglobina $\left(\mathrm{SaO}_{2}\right)$ e potencial de hidrogênio $(\mathrm{pH})$ em gatas submetidas à ovariosalpingohisterectomia sob infusão contínua de propofol, com dexmedetomidina (GDEX) ou solução salina via epidural (GSAL).

\begin{tabular}{|c|c|c|c|c|c|c|c|c|}
\hline Variável & Grupo & $\mathrm{T}-15$ & T0 & $\mathrm{T} 5$ & $\mathrm{~T} 10$ & $\mathrm{~T} 20$ & $\mathrm{~T} 30$ & $\mathrm{~T} 40$ \\
\hline \multirow{2}{*}{$\begin{array}{l}\text { PAM } \\
(\mathrm{mmHg})\end{array}$} & GDEX & $145 \pm 26$ & $109 \pm 12 \mathrm{~A}$ & $95 \pm 26 \mathrm{~A}$ & $160 \pm 13$ & $141 \pm 24$ & $129 \pm 26$ & $118 \pm 28$ \\
\hline & GSAL & $137 \pm 20$ & $123 \pm 28$ & $115 \pm 35$ & $165 \pm 11 \mathrm{~A}$ & $136 \pm 23$ & $129 \pm 11$ & $132 \pm 12$ \\
\hline \multirow{2}{*}{$\begin{array}{l}\text { PAS } \\
(\mathrm{mmHg})\end{array}$} & GDEX & $173 \pm 17$ & $145 \pm 12$ & $123 \pm 24 \mathrm{~A}$ & $187 \pm 17$ & $161 \pm 19$ & $147 \pm 34$ & $144 \pm 29$ \\
\hline & GSAL & $165 \pm 22$ & $147 \pm 34$ & $136 \pm 35$ & $192 \pm 19 A$ & $154 \pm 20$ & $151 \pm 14$ & $149 \pm 18$ \\
\hline \multirow{2}{*}{$\begin{array}{l}\text { PAD } \\
(\mathrm{mmHg})\end{array}$} & GDEX & $119 \pm 20$ & $101 \pm 14$ & $80 \pm 23 \mathrm{~A}$ & $141 \pm 10$ & $124 \pm 16$ & $111 \pm 22$ & $108 \pm 21$ \\
\hline & GSAL & $121 \pm 12$ & $110 \pm 27$ & $98 \pm 37$ & $142 \pm 11$ & $123 \pm 24$ & $119 \pm 16$ & $117 \pm 9$ \\
\hline \multirow{2}{*}{$\begin{array}{l}\text { FC } \\
\left(\text { bat } \min ^{-1}\right)\end{array}$} & GDEX & $188 \pm 29$ & $173 \pm 36$ & $178 \pm 32$ & $179 \pm 24$ & $170 \pm 19$ & $158 \pm 10$ & $149 \pm 20 \mathrm{a}$ \\
\hline & GSAL & $186 \pm 28$ & $184 \pm 27$ & $171 \pm 44$ & $183 \pm 30$ & $177 \pm 23$ & $176 \pm 28$ & $184 \pm 32 b$ \\
\hline \multirow{2}{*}{$\begin{array}{l}\mathrm{f} \\
\left(\operatorname{mov} \mathrm{min}^{-1}\right)\end{array}$} & GDEX & $35 \pm 10$ & $18 \pm 9$ & $20 \pm 3 \mathrm{~A}$ & $16 \pm 3 \mathrm{~A}$ & $18 \pm 2 \mathrm{Aa}$ & $20 \pm 3 \mathrm{Aa}$ & $20 \pm 3 \mathrm{~A}$ \\
\hline & GSAL & $54 \pm 10$ & $36 \pm 14$ & $22 \pm 7 \mathrm{~A}$ & $24 \pm 7 \mathrm{~A}$ & $24 \pm 6 \mathrm{Ab}$ & $24 \pm 5 \mathrm{Ab}$ & $30 \pm 12 \mathrm{~A}$ \\
\hline \multirow{2}{*}{$\begin{array}{l}\mathrm{PaCO}_{2} \\
(\mathrm{mmHg})\end{array}$} & GDEX & $26,3 \pm 3$ & $31,1 \pm 4$ & $44,2 \pm 7 \mathrm{~A}$ & $46,2 \pm 8 \mathrm{~A}$ & $44,1 \pm 5 \mathrm{~A}$ & $43,7 \pm 8 \mathrm{~A}$ & $39,8 \pm 5 \mathrm{Aa}$ \\
\hline & GSAL & $27,0 \pm 2$ & $30,2 \pm 3$ & $35,8 \pm 10 \mathrm{~A}$ & $42,6 \pm 12 \mathrm{~A}$ & $37,1 \pm 6 \mathrm{~A}$ & $34,4 \pm 6$ & $28,9 \pm 5 b$ \\
\hline \multirow{2}{*}{$\begin{array}{c}\mathrm{PaO}_{2} \\
(\mathrm{mmHg})\end{array}$} & GDEX & $98,7 \pm 4$ & $90,6 \pm 4$ & $404,1 \pm 42 \mathrm{~A}$ & $507,8 \pm 32 \mathrm{~A}$ & $510,4 \pm 30 \mathrm{~A}$ & $457,3 \pm 99 \mathrm{~A}$ & $495,6 \pm 46 \mathrm{~A}$ \\
\hline & GSAL & $97,4 \pm 5$ & $89,7 \pm 13$ & $378,9 \pm 82 \mathrm{~A}$ & $483,4 \pm 78 \mathrm{~A}$ & $466,9 \pm 75 \mathrm{~A}$ & $464,7 \pm 101 \mathrm{~A}$ & $403,6 \pm 186 \mathrm{~A}$ \\
\hline \multirow[t]{2}{*}{$\mathrm{SaO}_{2}(\%)$} & GDEX & $97,5 \pm 0,8$ & $97,1 \pm 0,9$ & $99,0 \pm 0,6 \mathrm{~A}$ & $99,3 \pm 0,5 \mathrm{~A}$ & $99,5 \pm 0,5 \mathrm{~A}$ & $98,8 \pm 0,9 \mathrm{~A}$ & $99,1 \pm 0,7 \mathrm{~A}$ \\
\hline & GSAL & $97,1 \pm 0,7$ & $96,1 \pm 1,7$ & $99,0 \pm 1 \mathrm{~A}$ & $99,5 \pm 0,5 \mathrm{~A}$ & $99,8 \pm 0,4 \mathrm{~A}$ & $99,6 \pm 0,5 \mathrm{~A}$ & $99,3 \pm 0,8 \mathrm{~A}$ \\
\hline \multirow[t]{2}{*}{$\mathrm{pH}$} & GDEX & $7,41 \pm 0,04$ & $7,39 \pm 0,04$ & $7,28 \pm 0,04 \mathrm{~A}$ & $7,27 \pm 0,03 \mathrm{~A}$ & $7,27 \pm 0,02 \mathrm{~A}$ & $7,27 \pm 0,01 \mathrm{Aa}$ & $7,30 \pm 0,04 \mathrm{Aa}$ \\
\hline & GSAL & $7,40 \pm 0,04$ & $7,37 \pm 0,04$ & $7,28 \pm 0,04 \mathrm{~A}$ & $7,28 \pm 0,03 \mathrm{~A}$ & $7,30 \pm 0,02 \mathrm{~A}$ & $7,33 \pm 0,05 \mathrm{Ab}$ & $7,37 \pm 0,04 b$ \\
\hline
\end{tabular}

Letra A na linha indica valor diferente de $\mathrm{T}-15(\mathrm{P}=0,05)$.

Letra minúscula diferente nas colunas, dentro de cada variável, indica valor diferente entre grupos $(\mathrm{P}=0,05)$.

quando o fármaco é utilizado como agente único (SMITH et al., 1993).

A TR foi menor de T20 até T40, no GDEX, e em T30 e T40, no GSAL. Da mesma forma, FERRO et al. (2005), com infusão contínua de propofol em cães, nas doses de 0,$2 ; 0,4$ e $0,8 \mathrm{mg} \mathrm{kg}^{-1} \mathrm{~min}^{-1}$, observaram redução da temperatura retal sem alteração da FC.

O tempo de extubação foi de 27,3 minutos no GSAL e de 27,5 minutos no GDEX. Não foram observadas diferenças significativas na FC, fe TR entre os grupos no período pós-operatório imediato, com seis horas de avaliação. Quanto à recuperação, 50\% (3/6) dos animais do GSAL apresentaram agressividade, enquanto que no GDEX 16,6\% (1/6) dos animais tiveram o mesmo comportamento. Em relação à intensidade de dor ao toque da ferida, 33,3\% (2/6) dos animais do GSAL receberam escore 0 (sem reação), 33,3\% (2/6) escore 1 (reação leve) e 33,3\% (2/6) escore 2 (reação intensa). No GDEX, 33,3\% (2/6) dos animais receberam escore 2 (reação intensa) e 66,7\% (4/6) receberam escore 0 (sem reação). A recuperação anestésica é freqüentemente acompanhada de dor decorrente do estímulo cirúrgico, estresse, aumento da concentração plasmática de catecolaminas, aumento da FC e pressão arterial e depressão respiratória. Desse modo, os efeitos analgésicos da dexmedetomidina podem trazer benefícios ao período de recuperação anestésica (GELTLER etal., 2001).

\section{CONCLUSÃO}

Conclui-se que os animais que receberam dexmedetomidina pela via epidural apresentaram plano de anestesia mais estável, com melhor grau de analgesia no período trans e pós-operatório, recuperação anestésica de melhor qualidade, sem alterações cardiovasculares e hemogasométricas significativas. 


\section{AGRADECIMENTOS}

À Universidade do Estado de Santa Catarina (UDESC), pela concessão de bolsa de Iniciação Científica, modalidade PROBIC.

\section{COMITÊ DE ÉTICA E BIOSSEGURANÇA}

Este estudo foi aprovado pela Comissão de Ética e Bem Estar Animal (CETEA) da Universidade do Estado de Santa Catarina, protocolo no $1.14 / 06$.

\section{FONTES DE AQUISIÇÃO}

a - Sevocris, Cristália Produtos Químicos e Farmacêuticos Ltda Itapira, SP, Brasil.

b - Ketamin S(+), Cristália Produtos Químicos e Farmacêuticos Ltda Itapira, SP, Brasil.

c - Dormire, Cristália Produtos Químicos e Farmacêuticos Ltda Itapira, SP, Brasil.

d - Propofol, Cristália Produtos Químicos e Farmacêuticos Ltda Itapira, SP, Brasil.

e - Precedex (100mcg ml-1), Abbott, SP, Brasil

f - Tranil, Hipolabor Farmacêutica Ltda, Sabará, MG, Brasil

\section{REFERÊNCIAS}

BAGATINI, A. et al. Dexmedetomidina: farmacologia e uso clínico. Rev Bras Anestesiol, v.52, n.5, p.606-617, 2002. Disponível em: http://www.scielo.br/scielo.php?script=sci_arttext\&pid=S0034$70942002000500012 \& \operatorname{lng}=$ en\&nrm=iso\&tlng=pt. Doi: $10.1590 /$ S0034-70942002000500012.

BLOOR, B.C. et al. Hemodynamic and sedative effects of dexmedetomidine in dog. J Pharmacol. Exp Therap, v.263, n.2, p.690-697, 1992.

BOUILLON, T.W. et al. Phatmacodynamic interaction between propofol and remifentanil regarding hypnosis, tolerance of laryngoscopy, bispectral index and electroencephalographic approximate entropy. Anesthesiol, v.100, p.1353-72, 2004.

CASTRO, V.B. Avaliação dos efeitos analgésicos e cardiovasculares da infusão contínua de propofol e cloridrato de dexmedetomidina em felinos. 2005. $131 \mathrm{f}$. Dissertação de Mestrado em Anestesiologia. Curso de Pósgraduação em Cirurgia, Universidade Estadual Paulista. Faculdade de Medicina de Botucatu.

CORTOPASSI, S.R.G.; FANTONI, D.T. Medicação préanestésica In: CORTOPASSI, S.R.G.; FANTONI, D.T. Anestesia em cães e gatos. São Paulo: Roca, 2002. Cap.13, p. $151-158$.

EISENACH, J.C. et al. Alpha ${ }_{2}$ - adrenergic agonistic for regional anesthesia. Anesthesiol, v.85, p.655-674, 1996.

FERRO, P. C. et al. Variáveis fisiológicas em cães submetidos à infusão contínua de diferentes doses de propofol. Ciênc Rural, v.35, n.5, p.1103-1108, 2005. Disponível em: http:// www.scielo.br/scielo.php? script $=$ sci_arttext\&pid $=\mathrm{S} 0103$ $84782005000500018 \& \operatorname{lng}=$ en $\& n r m=$ iso\&tlng=pt. Doi: $10.1590 / \mathrm{S} 0103-84782005000500018$.
FUKUSHIMA, K. et al. The effects of epidural administered dexmedetomidine on central and peripheral nervous system in man. Anesth analg, v.84, p.292-298, 1997.

GABAS, D.T. et al. Estudo clínico e cardiorrespiratório em cadelas gestantes com parto normal ou submetidas à cesariana sob anestesia inalatória com sevofluorano. Arq Bras Med Vet Zootec, v.58, n.4, p.518-524, 2006. Disponível em: http:// www.scielo.br/scielo.php?script $=$ sci_arttext\&pid $=$ S0102$09352006000400011 \& \operatorname{lng}=\mathrm{en} \& \mathrm{nrm}=\overline{\mathrm{is}} \& \mathrm{t} \operatorname{lng}=\mathrm{pt}$. Doi: 10.1590/S0102-09352006000400011.

GELTLER, R. et al. Dexmedetomidine a novel sedativeanalgesic agent. Proc (Bayl Univ Med Cent) v.14, n.1, p.1321,2001

HELLYER, P.W. et al. Induction of anesthesia with diazepamketamine and midazolam-ketamine in greyhounds. Vet Surg, v.20, n.2, p.143-147, 1991. Disponível em: http:// www3.interscience.wiley.com/journal/119842698/ abstract?CRETRY $=1 \&$ SRETRY $=0$. Doi: $10.1111 / \mathrm{j} .1532-$ 950X.1991.tb00324.x.

HONARMAND, A.; SAFAVI, M. Prevention of propofolinduced injection pain by sufentanil: a placebo-controlled comparison with remifentanil. Clin Drug Invest, v.28, n.1, p.27-35, 2008.

ILKIW, J.E. et al. The behaviour of healthy awake cats following intravenous and intramuscular administration of midazolan. $\mathbf{J}$ Vet Pharmacol Therap, v.19, n.3, p.205-216, 1996.

ILKIW, J.E. et al. The optimal intravenous dose of midazolam after intravenous ketamine in healthy cats. J Vet Pharmacol Therap, v.21, p.54-61, 1998. Disponível em: http:// www3.interscience.wiley.com/journal/119116106/abstract. Doi: 10.1046/j.1365-2885.1998.00102.x.

FUJII, Y.; ITAKURA, M. Comparison of lidocaine, metoclopramide, and flurbiprofen axetil for reducing pain on injection of propofol in Japanese adult surgical patients: a prospective, randomized, double-blind, parallel-group, placebocontrolled study. Clin Therap, v.30, n.2, p.280-286, 2008. Disponível em: http://www.sciencedirect.com/ science?_ob=ArticleURL\&_udi=B6VRS-4S21K0D$6 \&$ \& us e r $=687358 \&$ \& d o c $=1 \&$ _f m t $=\&$ _orig 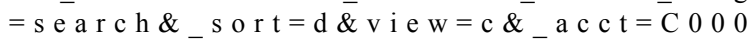 $037899 \&$ \& version $=1 \&$ \&_urlVersion $=0 \&$ \&_userid $=6$ $87358 \& \mathrm{md} \overline{5}=\mathrm{fd} 3833 \mathrm{c} 91 \mathrm{ad} \overline{7} 6202 \mathrm{bdf} 7 \mathrm{~b} 877 \mathrm{ea} 2 \mathrm{f} 4 \mathrm{bc} 1$. Doi: 10.1016/j.clinthera.2008.02.018.

KAYA, S et al. Lidocaine for prevention of propofol injectioninduced pain: a prospective, randomized, double-blind, controlled study of the effect of duration of venous occlusion with a tourniquet in adults. C Therap Res, v.69, n.1, p.29-35, 2008. Disónível em: http://www.sciencedirect.com/ science?_ob=ArticleURL\&_udi=B6VS 8-4S62 MFF$4 \&$ _ u s e r $=6873558 \&_{-} \mathrm{rd} \mathrm{o} \mathrm{c}=1 \& \&_{-} \mathrm{fm}$ $\mathrm{t}=\&$ orig $=$ search $\&$ sort $=\overline{\mathrm{d}} \& \mathrm{view}=\mathrm{c} \&$ ac ct $=$ C $000037899 \&$ \& version $=1 \&$ \&_ur 1 Versio $\bar{n}=0 \&$ _ userid $=687358 \&$ md $5=823$ ee 2 e 6 ac 9 ea 33702 bbe232e989ceb9. Doi: 10.1016/j.curtheres.2008.02.005.

KUUSELA, E. et al. Comparision of medetomidine and dexmedetomidine as premedicants in dogs undergoing propofol- 
isoflurane anestesia. Am J Vet Res, v.62, n.7, p.1073-1080, 2001.

LIN, H.C. Dissociative anesthetics. In: LUMB, W.V.; JONES, E.W. Veterinary anesthesia. 3.ed. Baltimore: Williams \& Wilkins, 1996. Cap.10, p.241-296.

MUIR, W.W.; GADAWSKI, J.E. Cardiovascular effects of a high dose of romifidine in propofol-anesthetized cats. Am J Vet Res v.63, n.9, p.1241-1246, 2002. Disponível em: http:/ /avmajournals.avma.org/doi/abs/10.2460/ajvr.2002.63.1241. Doi: $10.2460 /$ ajvr.2002.63.1241

OTERO, P.E. Dor: avaliação e tratamento. São Paulo: Interbook, 2005. 293p.

PADDLEFORD, R.R. Drogas anestésicas. In: PADDLEFORD R.R. Manual de anestesia em pequenos animais. 2.ed. São Paulo: Roca, 2001. Cap.3, p.37-88.

PASCOE, P.J. et al. The effect of the duration of propofol administration on recovery from anesthesia in cats. Vet Anaesth Analg v.33, p.2-7, 2006. Disponível em: http:// www3.interscience.wiley.com/journal/118606791/abstract. Doi: 10.1111/j.1467-2995.2005.00216.x.

ROBERTSON, S.A. Assessement and management of acute pain in cats. J Vet Emerg and Crit Care, v.15, n.4, p.261$272,2005$.

SELMI, A. et al. Infusão contínua de propofol em gatos prémedicados com cetamina-midazolam. Arq Bras de Med Vet e Zoot, v.57, n3, p.295-299, 2005. Disponível em: http:// www.scielo.br/scielo.php? script $=$ sci_arttext\&pid $=$ S010209352005000300003\&lng=en\&nrm=iso\&tlng=pt. Doi: $10.1590 / \mathrm{S} 0102-09352005000300003$.
SKARDA, R. Local and regional anesthetic and analgesic techinique: dogs. In.: TRANQUILLI, W.J.; BENSON, G.J. Lumb and Jones' veterinary anesthesia. Baltimore: Williams and Wilkins, 1996. p.434-447.

SMITH, J.A. et al. Adverses effects of administration of propofol with various preanesthetic regimens in dogs. J Am Vet Med Assoc, v.202, n.7, p.1111-1115, 1993.

SOUZA, S.S. Efeitos da dexmedetomidina, por via epidural ou infusão contínua intravenosa, em gatas anestesiadas com propofol e isofluorano e submetidas a ovariossalpingohisterectomia. 2006. 140f. Dissertação (Mestrado em Medicina Veterinária) - Faculdade de Medicina Veterinária e Zootecnia, USP.

VILLELA, N.R.; JUNIOR, P.N. Uso de dexmedetomidina em anestesiologia. Rev Bras Anest, v.53, n1, p.97-113, 2003. Disponível em: http:/www.scielo.br/scielo.php?script=sci_arttext\&pid=S0034$70942003000100013 \& \operatorname{lng}=$ en\&nrm=iso\&tlng=pt. Doi: $10.1590 /$ S0034-70942003000100013.

TORSKE, K.E. et al. End tidal halothane concetration and postoperative analgesia requirements in dogs: A comparison between intravenous oxymorphone and epidural bupivacaine alone and in combination with oxymorphone. Can Vet $\mathbf{J}$ v.39, p.361-369, 1998

WEITZ, J.D. et al. Anesthetic and hemodynamic effects of dexmedetomidine during isoflurano anesthesia in a canine model. N Anesth, v.2, n.1, p.19-27, 1991.

YANG, C.Y. et al. Propofol inhibits medullary pressor mechanisms in cats. C J Anesth, v.44, n.7, p.775-781, 1997. 